\title{
Rotor apparatus performance in the production of graphene-containing suspensions
}

\author{
Ruaa A. Al-Jarah ${ }^{1}$, Andrey Baranov ${ }^{2}$, and Anastasiya Pasko $^{2}$ \\ ${ }^{1}$ Post-graduate student from the Republic of Iraq, Tambov State Technical University, 106 \\ Sovetskaya Street, 392000, Tambov, Russian Federation \\ ${ }^{2}$ Department of Technology and Methods of Nanoproducts Manufacturing, Tambov State Technical \\ University, 106 Sovetskaya Street, 392000, Tambov, Russian Federation
}

\begin{abstract}
A new design of the rotor apparatus and the order of the experiments are proposed, which allows you to change individual parameters and study their influence on the intensity of the process. It has been theoretically and experimentally proved that with a change in the volume of the suspension passing through the rotor apparatus per unit time, the performance on graphene structures does not change. It is necessary to increase productivity either by increasing the rotor speed, or by increasing the concentration of graphite in the initial suspension.
\end{abstract}

\section{Introduction}

Graphene is one of the most promising materials of our time due to its unique properties [1]. One of the most remarkable advantages of graphene is that even in very small quantities it can greatly improve the performance properties of structural and functional materials. For example, adding only 0.07 weight percent of few-layer graphene to concrete increases the compressive and bending strengths by 146 and 79 percent, respectively [2]. In addition, a reduction in water permeability of almost $400 \%$ compared with conventional concrete makes this new composite material ideal for construction in areas prone to flooding. It has been experimentally established that the three-point bending strength of the graphene-modified samples (graphene concentration of about $0.05 \mathrm{wt} . \%$ with respect to cement) is about 1.5 times greater than that of the pristine concrete, whereas the water absorption of the 2DCM-modified concrete decreases by 3 times. Using the method for modifying the epoxy resin with the 2DCM the Charpy impact strength is increased by 25 $\%$. The tensile and bending strength increase by $10 \%$. The test results of the repaired beams after breaking at three-point bending, as well as on the adhesion of the reinforcing fabric to the concrete, revealed a synergistic effect while using the 2DCM-modified concrete and epoxy resin [3]. The review [4] details the methods for modifying epoxy resin with fewlayer graphene.

Few-layer and multilayer graphene is used to improve the tribological characteristics of lubricants [5-8]. The paper [5] describes the results of a study of the graphene concentration effect on the viscosity of base oil for preparing plastic lubricants. It was established that the

*Corresponding author:barr2@yandex.ru 
viscosity of the graphene masterbatch reaches the viscosity of the plastic lubricant at 9-11 $\mathrm{wt} \%$ for few-layered graphene and $5-8 \mathrm{wt} \%$ for multi-layered one. It was found that if graphite is peeled off in the base oil used for the production of lubricant, the friction coefficient and the diameter of the wear spot are reduced by 35 and $25 \%$, respectively, due to the modification of the lubricant with $0.12 \mathrm{wt} . \%$ few-layer graphene [6]. Modification with $0.1 \%$ multilayer graphene complex calcium grease, the diameter of the wear spot decreased by $50 \%$, the scoring index increased 2.9 times, and the bearing capacity increased 3.8 times [7].

As a result of processing graphite lubricant in a planetary mill, the friction coefficient decreased by 3 times [9]. For the industrial production of graphene concentrates, which can modify plastic lubricants, a technology is proposed that is implemented in a drum rod mill [10-12]. Graphene nanoparticles can be obtained from expanded graphite compounds by mechanochemical synthesis [13].

The problems of the modification of lubricants by nanoparticles, including graphene, are considered in detail in a review [14].

\section{Experiments}

The authors [2] used the Silverson model L5M mixer, which generates high shear due to the closely spaced $(0.1 \mathrm{~mm})$ rotor / stator combination. The experiments were carried out as follows. A fixed amount of crystalline graphite (powder) was poured into 4.5 liters of water, which was in a cylindrical container. To prevent aggregation of graphene plates, Nmethyl-2-pyrrolidone (NMP) or sodium cholate $(\mathrm{NaC})$ was added to water. The stator and rotor were placed in a container and the rotor began to rotate at a speed $4500 \mathrm{rpm}$. Suspension due to centrifugal forces, which creates a rapidly rotating rotor, is ejected through holes in the side surface of the stator. In the zone between the rotor and the stator, a vacuum (vacuum) is created and the suspension is sucked in through the upper and lower ends of the stator. Thus, the mixer works like a pump. After centrifugation, these suspensions contained a large number of high-quality graphene nanosheets, including some monolayers. It was experimentally established that exfoliation takes place only if the gap between the stator and rotor blades is less than $0.1 \mathrm{~mm}$ and the shear rate is more than $10^{4} \mathrm{~s}^{-}$ 1 .

In our opinion, this device design has several disadvantages. First of all, the fluid motion is random in nature. Despite the fact that there is a circulation circuit there is no guarantee that all particles of graphite, for a fixed period of time, will pass through the holes in the stator the same number of times. Changing the position of the mixer in the tank we obtained different results, in particular, different concentrations of graphene nanostructures in suspension after the same centrifugation regimes. In addition, during prolonged use, the blades and the inner surface of the stator are abraded and the gap between them increases. So, after 700 hours of operation, the exfoliation of graphite particles practically stopped, probably because the gap became more than $0.1 \mathrm{~mm}$.

The design of a rotary apparatus with moving blades was previously proposed [15], and experiments were performed as follows. We prepared $3 \ldots 6$ liters of oil suspension of crystalline fuel GSM-2 with a concentration of $2.5-10 \%$. The suspension was poured into a cylindrical container, a rotary apparatus was installed in this container, and processing of the suspension was started. Every 5 cycles of suspension processing, samples were taken to determine the concentration of graphene structures.

During rotation of the rotor, the centrifugal forces acted on the suspension, which ejected the suspension through the holes in the stator of the apparatus. In the zone between the rotor and the stator a rarefied state (vacuum) was formed and new portions of the suspension were absorbed into this zone. In fact, the rotary apparatus worked like a pump. 
The suspension in the container moved along a closed circulation loop periodically passing through the apparatus where graphite exfoliated. The dependence of changes in the concentration of graphene structures (low-layer and multilayer graphene) in suspension on the main parameters was experimentally obtained: the concentration of graphite in the initial suspension is $c_{O}$; rotor speed; stator inner radius $R$; the number of blades is $n$; processing time $t$. The concentration of graphene structures depends almost linearly on all these parameters:

$$
c=k c_{L}\left[\left(c_{O} / c_{L O}\right) \times\left(R / R_{L}\right) \times\left(\omega / \omega_{L}\right) \times\left(n / n_{L}\right) \times\left(t / t_{L}\right)\right]
$$

where $c$ is the concentration of graphene structures in the designed apparatus, the index $L-$ refers to a laboratory setup.

Thus, in order to calculate the concentration of graphene structures that can be obtained in the designed rotary apparatus, it is necessary to experimentally determine the numerical value of the concentration $c_{L}$ in a laboratory setup and then calculate the value of $c$ from dependence (1).

In the transition from laboratory installations to industrial apparatus, it is advisable to switch from a batch process to a continuous one. Figure 1 shows a diagram of the rotary apparatus that we used in this work. A rotor 2 is located in the cylindrical stator 1 . The suspension is fed into the apparatus through the nozzle 3 and leaves the apparatus through the nozzle 4 . The rotor 2 has radial grooves in which the movable blades 5 are mounted. When the rotor rotates, under the influence of centrifugal forces, the blades 5 are pressed against the inner surface stator 1 and slide on it without a gap. A roof 6 was installed on top of the stator 1 , and a bottom 7 was installed on the bottom.
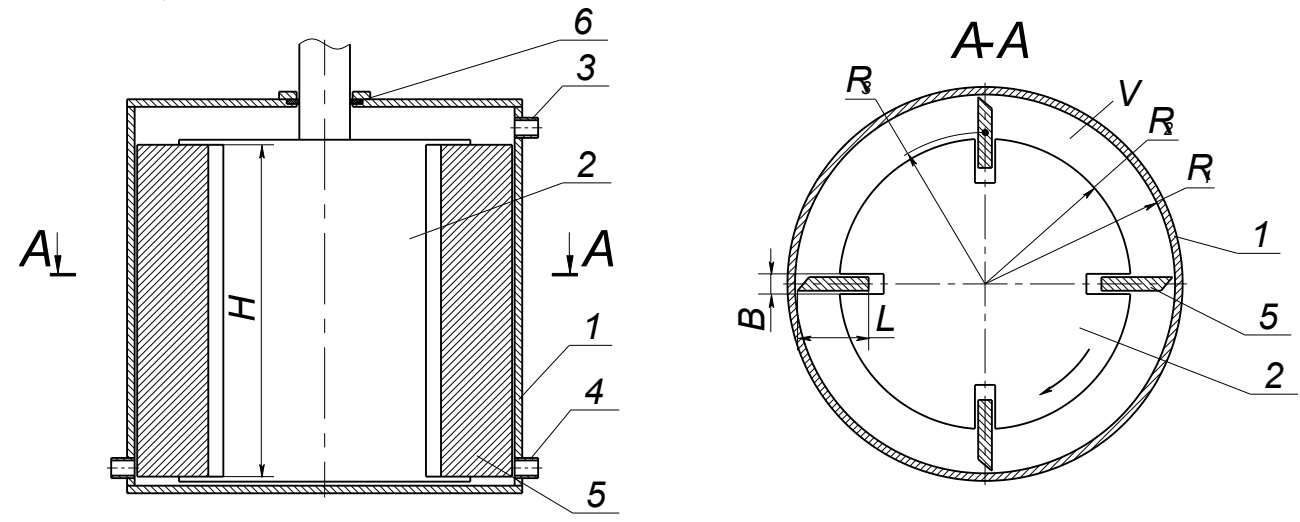

Fig.1. Scheme of the rotary apparatus.

The general installation diagram, which is shown in figure 2, was also changed.

From 3 to 5 liters of liquid was poured into a container 1 and crystalline graphite powder. The concentration of graphite in the initial suspension ranged from 2.5 to $20 \%$. The liquid used was water or synthetic oil. The paddle mixer installed in the container 1 prevented the deposition of graphite to the bottom. After preparing the initial suspension, the valve $V_{1}$ was opened and the suspension was supplied to the rotary apparatus 2 using pump $P_{1}$. After all the suspension from the container 1 passes through the rotary apparatus and moves into the container 3 , we closed the valve $V_{1}$, turned off the pump $P_{1}$ and the rotary apparatus 2 . One cycle of processing the suspension was completed. 


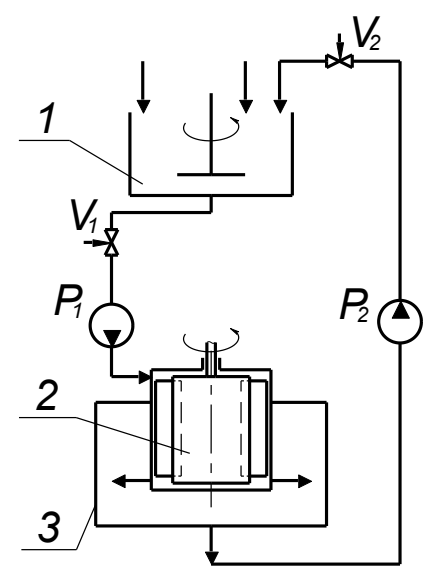

Fig. 2. Schematic diagram of the installation for liquid-phase shear exfoliation of graphite.

Then we opened the valve, turned on the pump and the suspension passed from container 3 to container 1 . After turning off the pump and closing the valve, the device is ready for the next suspension treatment cycle.

Next, open the valve, turn on the pump and move the suspension from container 3 to container 1 . After turning off the pump and closing the valve, the unit is ready for the next suspension treatment cycle.

In the experiments, we changed the flow rate of the suspension from 5 to 10 liters per minute, and the rotor speed from 5000 to $15000 \mathrm{rpm}$.

Every 30 cycles of suspension processing, samples were taken to determine the concentration of graphene structures.

\section{Results and discussion}

Figure 3 shows the dependence of the concentration of graphene structures on the number of suspension processing cycles. The rotor speed was $10,000 \mathrm{rpm}$, the graphite concentration in the initial suspension was $50 \mathrm{mg} / \mathrm{ml}$, suspension volume was 5 liters and pump $P$ was 5 liters per minute.

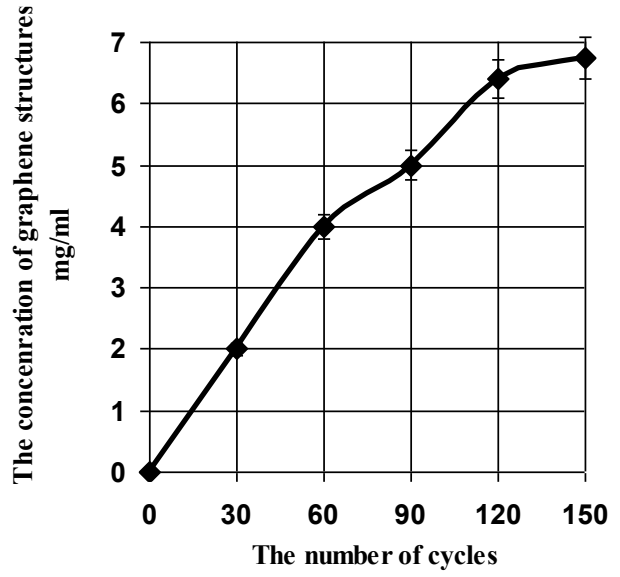

Fig. 3. Dependence of the concentration of graphene structures on the number of suspension processing cycles. 
Comparing the results obtained earlier with the results presented in figure 2, we saw that previously the maximum concentration of graphene structures in oil was achieved in 25 cycles [16], and in the present work for 150 cycles. Assuming that such a big difference arose because of different experimental methods, we calculated the time of one cycle for these two options for exfoliation. In the first case, the time of one cycle was taken equal to $300 \mathrm{~s}$, and in our case, the time of one cycle (the time during which the entire suspension passes through the rotary apparatus) is $60 \mathrm{~s}$. Thus, in [16], the maximum concentration of graphene structures in the suspension was achieved in $125 \mathrm{~s}$, and in our case, in $90 \mathrm{~s}$. It is likely that such a reduction in processing time occurred because in our case the entire suspension passes through the apparatus only once. With a periodic process, it is possible that part of the suspension does not enter the apparatus during one cycle.

It was experimentally established that with an increase or decrease in the rotor speed, the change in the concentration of graphene structures in one processing cycle did not change. In order to explain this fact, we determine the length of the path that a graphite particle travels in a rotary apparatus in one exfoliation cycle. First of all, we calculated the volume between the rotor and the stator (Figure 1):

$$
V=\pi H\left(R_{1}^{2}-R_{2}^{2}\right)=3.14 \cdot 70 \cdot\left(20^{2}-18.5^{2}\right)=12693 \mathrm{~mm}^{3} .
$$

Given that the pump capacity is equal to $5,000,000 \mathrm{~mm}^{3}$, the time of one cycle will be equal to:

$$
t=V / Q=126993 / 5000000=0.015 \mathrm{~s} .
$$

The length $L_{1}$ of the sliding path of the particle along the inner surface of the rotor will be equal to:

$$
L_{1}=2 \pi R_{1} n t
$$

where $n$ is the rotor speed of revolutions per second.

Particular attention should be paid to the fact that if we increase productivity $Q$, then the residence time $t$ of particles in the apparatus will be proportionally reduced, i.e. in the exfoliation zone. As a result, the increase in the concentration of graphene structures in the suspension will decrease. However, at the same time, the time of one cycle will be proportionally reduced. In the final result, an increase in the concentration of graphene structures per unit time will not change. If, at a constant value of productivity, the rotor speed is increased, the path length in one cycle will increase and, as a result, the concentration of graphene structures will increase.

In the periodic mode, it was not taken into account that with an increase in the rotor speed, the volume of the suspension increases, which passes through the rotor apparatus per unit time, which leads to a decrease in the time for which one exfoliation cycle takes place. The presented methodology for the exfoliation process (figure 2) allows you to independently change the process parameters and find the real influence of these parameters on performance.

\section{Conclusion}

A new design of the rotor apparatus and the order of the experiments are proposed, which allows you to change individual parameters and study their influence on the intensity of the process. It has been theoretically and experimentally proved that with a change in the volume of the suspension passing through the rotor apparatus per unit time, the performance on graphene structures does not change. It is necessary to increase 
productivity either by increasing the rotor speed, or by increasing the concentration of graphite in the initial suspension.

\section{References}

1. A.P. Kauling, A.T. Seefeldt, D.P. Pisoni, R.C. Pradeep, R. Bentini, R.V.B. Oliveira, K.S. Novoselov, A.H.C. Neto, Adv. Mater. 30, 03784 (2018)

2. D. Dimov, I. Amit, O. Gorrie, M.D. Barnes, N.J. Townsend, A.I. Neves, F. Withers, S. Russo, M.F. Craciun, Adv. Funct. Mater. 28, 1705183 (2018)

3. V. Pershin, K.A. Al-Shiblawi, A.M.R. Al-Mashhadani, A. Pasko, D. Melekhin, IOP Conf. Ser-Mat. Sci. 693(1), 012046 (2019)

4. K.A. Al-Shiblawi, V.F. Pershin, V.P. Yartsev, Mater. Sci. Forum 4, 42-53 (2018)

5. V. Pershin, K. Ovchinnikov, Z. Al-Hilo, N. Memetov, A. Tkachev, E. Galunin, AIP Conf. Proc. 2041, 020016 (2018)

6. V. Pershin, G. Zhumagalieva, A. Tkachev, A. Pasko, N. Memetov, J. Phys.Conf. Ser. 1278(1), 012025 (2019)

7. V.F. Pershin, K.A. Ovchinnikov, A.A. Alsilo, R.A. Stolyarov, N.R. Memetov, Nanotechnol. Russ. 13(5-6), 344-348 (2018)

8. V.F. Pershin, M.N. Krasnyanskiy, Z.A.A. Alhilo, A.M.R. Al-Mashhadani, A.A. Baranov, A.A. Osipov, IOP Conf. Ser.-Mat. Sci. 693(1), 012023 (2019)

9. D.A.Y. Al-Saadi, V.F. Pershin, B.N. Salimov, S.A. Montaev, J. Frict. Wear 38(5), 355358 (2017)

10. G. Zhumagalieva, V. Pershin, A. Tkachev, A. Vorobiev, A. Pasko, E. Galunin, AIP Conf. Proc. 2041, 020010 (2018)

11. V.F. Pershin, G.B. Zhumagaliyeva, A.G Tkachev, A.A. Pasko, A.M. Vorobyev, IOP Conf. Ser-Mat. Sci. 693(1), 012035 (2019)

12. A. Tkachev, G. Zhumagalieva, Z.A.A.A. Ali, N. Memetov, E. Galunin, V. Pershin, Modification of Frost-Resistant Plastic Lubricants Using Few- and Multi-Layered Graphene, in Proceedings of the 4th World Congress on Recent Advances in Nanotechnology (RAN'19), 14-16 April 2019, Rome, Italy (2019)

13. A.V. Melezhik, V.F. Pershin, N.R. Memetov, A.G. Tkachev, Nanotechnol. Russ. 11(78), 421-429 (2016)

14. I. Ali, Al A. Basheer, A. Kucherova, N. Memetov, T. Pasko, K. Ovchinnikov, V. Pershin, D. Kuznetsov, E. Galunin, V. Grachev, A. Tkachev, J Mol. Liq. 279 (1), 251 266 (2019)

15. K.A. Al-Shiblawi, A.A. Pasko, V.F. Pershin, Transactions TSTU, 24(4), 717-726 (2018)

16. V.F. Pershin, A.G. Tkachev, R.A. Al-Jarah, T.V. Pasko, A.A. Osipov, IOP Conf. Ser.Mat. Sci. 693(1), 012007 (2019) 